\title{
Severe Acute Respiratory Syndrome Coronavirus-2 Seropositivity in South-Central Uganda, During 2019 $-2021$
}

\author{
Charles Ssuuna ( $\nabla$ cssuuna@rhsp.org ) \\ Rakai Health Sciences Program \\ Ronald Moses Galiwango \\ Rakai Health Sciences Program
}

Edward Nelson Kankaka

Rakai Health Sciences Program

Joseph Kagaayi

Makerere University School of Public Health

Anthony Ndyanabo

Rakai Health Sciences Program

Godfrey Kigozi

Rakai Health Sciences Program

Gertrude Nakigozi

Rakai Health Sciences Program

Tom Lutalo

Uganda Virus Research Institute

Robert Ssekubugu

Rakai Health Sciences Program

John Bosco Wasswa

Rakai Health Sciences Program

Anthony Mayinja

Rakai Health Sciences Program

Martina Cathy Nakibuuka

Rakai Health Sciences Program

Samiri Jamiru

Rakai Health Sciences Program

John Baptist Oketch

Rakai Health Sciences Program

\section{Edward Muwanga}

Kyotera District Health Office, Ministry of Health

\section{Larry William Chang}


Department of International Health, Johns Hopkins Bloomberg School of Public Health, Baltimore, MD

\section{Mary Kate Grabowski}

Department of Epidemiology, Johns Hopkins Bloomberg School of Public Health, Baltimore, MD

\section{Maria Wawer}

Department of Epidemiology, Johns Hopkins Bloomberg School of Public Health, Baltimore, MD

\section{Ronald Gray}

Department of Epidemiology, Johns Hopkins Bloomberg School of Public Health, Baltimore, MD

\section{Mark Anderson}

Abbott Laboratories, Abbott Diagnostics Division, Abbott Park, Illinois

\section{Michael Stec}

Abbott Laboratories, Abbott Diagnostics Division, Abbott Park, Illinois

\section{Gavin Cloherty}

Abbott Laboratories, Abbott Diagnostics Division, Abbott Park, Illinois

\section{Oliver Laeyendecker}

Division of Infectious Disease, Division of Medicine, Johns Hopkins School of Medicine, Baltimore, MD

\section{Steven James Reynolds}

Division of Intramural Research, National Institute of Allergy and Infectious Diseases, National Institutes of Health, Bethesda, MD

\section{Thomas C. Quinn}

Division of Intramural Research, National Institute of Allergy and Infectious Diseases, National Institutes of Health, Bethesda, MD

\section{David Serwadda}

Makerere University School of Public Health

\section{Research Article}

Keywords: SARS-CoV-2 seroprevalence, healthcare workers, COVID-19, South-central Uganda

Posted Date: October 22nd, 2021

DOl: https://doi.org/10.21203/rs.3.rs-960585/v1

License: (9) This work is licensed under a Creative Commons Attribution 4.0 International License. Read Full License 


\section{Abstract}

Background: Globally, key subpopulations such as healthcare workers (HCWs) have a higher risk of contracting SARS-CoV-2. In Uganda, limited access to personal protective equipment amidst lack of clarity on the extent and pattern of the community disease burden may exacerbate this situation. We assessed SARS-CoV-2 antibody seroprevalence among high-risk sub-populations in South-central Uganda, including HCWs, persons within the general population previously reporting experiencing key COVID-19 like symptoms (fever, cough, loss of taste and smell) and archived plasma specimens collected between October 2019 - 18 ${ }^{\text {th }}$ March 2020, prior to confirmation of COVID-19 in Uganda.

Methods: From November 2020 - January 2021, we collected venous blood from HCWs at selected health facilities in South-Central Uganda and from population-cohort participants who reported specific COVID19 like symptoms in a prior phone-based survey conducted (between May to August 2020) during the first national lockdown. Pre-lockdown plasma collected (between October 2019 and March 18 ${ }^{\text {th }}, 2020$ ) from individuals considered high risk for SARS-CoV-2 infection was retrieved. Specimens were tested for

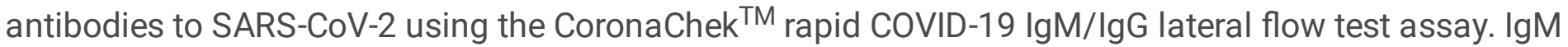
only positive samples were confirmed using a chemiluminescent microparticle immunoassay (CMIA) (Architect AdviseDx SARS-CoV-2 IgM) which targets the spike protein. SARS-CoV-2 exposure was defined as either confirmed IgM, both IgM and IgG or sole IgG positivity.

Results: The seroprevalence of antibodies to SARS-CoV-2 in HCWs was $21.1 \%$ [95\%Cl: 18.2-24.2]. Of the phone-based survey participants, $11.9 \%$ [95\% Cl: 8.0-16.8] had antibodies to SARS-CoV-2. Among 636 prelockdown plasma specimens, $1.7 \%$ [95\% Cl: 0.9-3.1] were reactive.

Conclusions: Findings suggest a high seroprevalence of antibodies to SARS-CoV-2 among HCWs and substantial exposure in persons presenting with specific COVID-19 like symptoms in the general population of South-central Uganda. Based on current limitations in serological test confirmation, it remains unclear whether pre-lockdown seropositivity implies prior SARS-CoV-2 exposure in Uganda.

\section{Background}

It is over a year since the Severe Acute Respiratory Syndrome Coronavirus 2 (SARS-CoV-2) emerged(1) as a global pandemic and as of the 2 nd of August 2021, nearly two hundred million cases were reported globally with $>4,000,000$ fatalities(2). Transmission occurs by respiratory droplets, aerosols, and via fomites and is higher in confined or congested spaces(3). SARS-CoV-2 infection can be asymptomatic(4) with estimates ranging from $5 \%-80 \%$ while symptoms are largely nonspecific and include features of flu-like illness(5). Diagnosis of asymptomatic and mild cases may be missed due to prioritization of screening/confirmatory tests for individuals with moderate to severe symptoms. However, asymptomatic and pre-symptomatic persons can be highly contagious and contribute greatly to epidemic spread $(6,7)$.

As of the 3rd of August 2021, more than 94,000 cases with 2,710 deaths were documented in Uganda(2). Community transmission is on the rise(8) despite earlier control measures that included a phased 
nationwide lockdown between March and August 2020(9). The SARS-CoV-2 diagnostic testing landscape in Uganda prioritizes testing for symptomatic persons. It is unknown how many infected asymptomatic persons are missed due to this symptom-based testing approach and what impact this has on community transmission.

HCWs in particular are at a higher risk of contracting SARS-CoV-2 $(10,11)$ and inadvertently transmitting it to their patients, some of whom may be immunocompromised. According to the World Health Organization (WHO), they account for $10 \%$ of the global SARS-CoV-2 burden(12). This risk may be higher in countries like Uganda, due to shortage of Personal Protective Equipment (PPE) amidst unquantified community disease burden. Notably, several HCWs in Uganda have been infected and a number have $\operatorname{died}(13)$.

Due to the limited testing capacity, there are likely to be many undetected community infections fueling the epidemic. It is also unknown if SARS-CoV-2 importation or exposure in Uganda might have occurred earlier than the first (official) case reported on the 21 st of March 2020. We aimed at determining the prevalence of antibodies to SARS-CoV-2 among selected high-risk sub-populations in South-central Uganda, including HCWs, persons who previously reported specific Coronavirus disease 2019 (COVID-19) like symptoms (fever, cough, loss of taste and smell) in the preceding 30 days, between May and August 2020. Additionally, we aimed at exploring the possibility of prior SARS-CoV-2 importation/exposure in South-Central Uganda before confirmation of the first (official) case on the 21st of March 2020.

\section{Methods}

\section{Study design and setting}

This study was cross-sectional and was conducted at the Rakai Health Sciences Program (RHSP) with participants recruited from within and outside the Rakai Community Cohort Study (RCCS) in four districts of South-central Uganda (Masaka, Kyotera, Rakai and Lyantonde). The RCCS is an open, populationbased cohort in 40 communities in these districts with surveys conducted every 18 months among 23,000 adults, resident in fishing, agrarian, or peri-urban/trading community settings(14).

\section{Study population and sample size}

A total of 980 participants including $753 \mathrm{HCWs}$ and 227 individuals from the RCCS phone-based survey were recruited into the study. Participants from the cohort had previously reported experiencing COVID-19 like symptoms (fever, cough, loss of taste and/or loss of smell) in the preceding 30 days during an earlier phone-based survey conducted between May and August 2020. HCWs were identified from health facilities in the region, prioritizing high volume facilities located near the Uganda-Tanzania border or along the Kampala-Mutukula highway serving mobile persons who may be at higher risk of SARS-CoV-2 acquisition. At the selected health facilities, all available, willing HCWs were recruited into the study. 
Additionally, we retrieved 636 archived plasma specimens collected between October 2019 and March 18th, 2020, before the first national lockdown took effect. Selected samples of persons living in RCCS communities close to the Tanzanian border and along the Kampala-Mutukula highway were considered to have a high risk for SARS-CoV-2 infection due to their high mobility and interaction with cross-border populations. They included traders/vendors, commercial sex work clients, fisher folks, bike (boda-boda) riders, truck drivers, mechanics, shopkeepers, and bar owners/workers.

\section{Sample / Data collection}

Participants in the phone-based survey conducted during the first lockdown and reported having previously experienced at least one of the above COVID-like symptoms were contacted for participation in this study. Additionally, study field teams approached HCWs at selected health facilities for participation. Consenting participants provided $4 \mathrm{mls}$ of venous blood specimens while a short questionnaire was administered to HCWs to collect data on participant demographics, cadre, prior SARS-CoV-2 exposure, and PPE access/use. Plasma was frozen $\left(-80^{\circ} \mathrm{C}\right)$ until laboratory analysis.

\section{Laboratory analysis}

Frozen plasma was thawed and tested for antibodies to SARS-CoV-2 using the CoronaChek ${ }^{\mathrm{TM}}$ rapid COVID-19 IgM/lgG lateral flow test assay as per manufacturer's instructions. This assay was previously validated with Ugandan samples, including 1077 pre-pandemic samples from the RCCS(15). Low specificities of SARS-CoV-2 antibody assays have been reported, particularly from malaria endemic regions $(16,17)$. Therefore, any sample that was solely $\operatorname{lgM}$ positive by CoronaChek ${ }^{\mathrm{TM}}$ was retested by the Abbott ARCHITECT AdviseDx SARS-CoV-2 IgM chemiluminescent microparticle immunoassay (CMIA) (Abbott, Chicago, IL).

\section{Data analysis}

SARS-CoV-2 exposure was defined as either IgM confirmed by the ARCHITECT CMIA assay, both IgM and IgG or IgG sole positivity. Point prevalence and $95 \%$ confidence intervals were determined for each subgroup using the exact Clopper-Pearson method of calculating confidence intervals for binomial proportions.

\section{Results}

Healthcare workers' SARS-CoV-2 antibody test results: Most of the participants were female (64.54\%) and were $25-34$ years of age (31.6\%). In the initial screening using the CoronaChek ${ }^{\mathrm{TM}}, 30.8 \%(232 / 753)$ of HCWs had detectable SARS-CoV-2 antibodies irrespective of isotype class. Of these, 119 tested positive for IgM only, 102 for both IgM and IgG and 11 for IgG only. Of the initially 119 IgM only reactive samples, 46 were confirmed positive when re-tested using the ARCHITECT assay. The overall seroprevalence of SARS-CoV-2 antibodies among HCWs was $21.1 \%$ [95\%Cl: 18.2-24.2] (159/753). Majority (24/26) of the 
sampled health facilities had at least one healthcare worker who had antibodies to SARS-CoV-2. Seropositivity was highest among nurses and lowest among medical officers (Table 1).

Table 1

Factors associated with SARS-CoV-2 seropositivity among Healthcare workers

\begin{tabular}{|llll|}
\hline Sociodemographic characteristics & $\begin{array}{l}\mathbf{n}(\%) \text { seropositive } \\
\mathbf{N}=159\end{array}$ & $\begin{array}{l}\text { Univariate } \\
\text { Odds Ratio (95\% Cl) }\end{array}$ \\
\hline Sex & Male & $91(38.4)$ & $0.9(0.6-1.2)$ \\
\hline Fge category & $15-24$ & $33(20.8)$ & $0.7(0.4-1.1)$ \\
\hline & $25-34$ & $57(35.8)$ & Ref. \\
\hline $35-44$ & $39(24.5)$ & $0.8(0.5-1.3)$ \\
\hline $45-54$ & $21(13.2)$ & $1.1(0.6-1.9)$ \\
\hline $55+$ & $9(5.7)$ & $0.7(0.3-1.5)$ \\
\hline Medical Officer & $3(1.9)$ & $1.3(0.4-5.1)$ \\
\hline Clinical Officer & $8(5.0)$ & $1.8(0.7-4.3)$ \\
\hline Nurse (all levels) & $57(35.8)$ & Ref \\
\hline Lab tech (all levels) & $16(10.1)$ & $1.4(0.8-2.7)$ \\
\hline Other staff & $75(47.2)$ & $1.0(0.7-1.5)$ \\
\hline
\end{tabular}

A total of $128 \mathrm{HCW}$ reported having undergone prior SARS-CoV-2 RT-PCR testing with 16 reporting a positive result. Of the 16 individuals, 8 had detectable antibodies to SARS-CoV-2. Out of the 128, a total of $108 \mathrm{HCW}$ reported previous negative RT-PCR results and $27 \%$ of these, subsequently tested antibody positive. Only face masks were reported to have been used by all HCWs who reported prior contact with a confirmed COVID-19 case. Despite reporting consistent use of face masks, $40 \%(63 / 156)$ of the HCWs reporting previous contact with a confirmed COVID-19 case had antibodies to SARS-CoV-2.

Cohort participants' SARS-CoV-2 antibody test results: Females comprised $69.1 \%$ and most participants were aged 35-44 years. Upon initial screening using the CoronaChek ${ }^{\mathrm{TM}}, 16.3 \%$ of the participants (37/227) tested positive on IgM only, 2.2\% (5/227) tested positive on IgG only whereas $6.6 \%(15 / 227)$ were positive on both IgM and IgG. Following retesting of the initially IgM only reactive samples using the ARCHITECT assay, 7/37 were confirmed positive. The overall seroprevalence of antibodies to SARS-CoV-2 in this population was $11.9 \%$ [95\% Cl: 8.0-16.8] (27/227). There was nearly no difference in seropositivity among HIV positive and negative participants (Table 2). 
Table 2

Factors associated with SARS-COV-2 seropositivity among phone-based survey participants

\begin{tabular}{|c|c|c|c|}
\hline \multicolumn{2}{|c|}{ Sociodemographic characteristics } & $\begin{array}{l}\text { n (\%) seropositive } \\
\mathrm{N}=27\end{array}$ & $\begin{array}{l}\text { Univariate } \\
\text { Odds Ratio }(95 \% \mathrm{Cl})\end{array}$ \\
\hline \multirow[t]{2}{*}{ HIV status } & Negative & $14(51.9)$ & Ref \\
\hline & Positive & $13(48.1)$ & $1.0(0.5-2.3)$ \\
\hline \multirow[t]{2}{*}{ Sex } & Male & $9(33.3)$ & $0.9(0.4-2.1)$ \\
\hline & Female & $18(66.7)$ & Ref \\
\hline \multirow[t]{4}{*}{ Age category } & $15-24$ & $0(0.0)$ & \\
\hline & $25-34$ & $7(25.9)$ & $0.9(0.4 .2 .4)$ \\
\hline & $35-44$ & $14(51.9)$ & Ref \\
\hline & $45-54$ & $6(22.2)$ & $1.5(0.5-4.2)$ \\
\hline \multirow[t]{9}{*}{ Occupation } & Agriculture for home use/barter & $10(37.0)$ & Ref \\
\hline & Agriculture for selling & $1(3.7)$ & $0.1(0.0-1.1)$ \\
\hline & Fishing & $3(11.1)$ & $1.2(0.3-4.8)$ \\
\hline & Shopkeeper & $3(11.1)$ & $1.5(0.4-6.7)$ \\
\hline & Trading/vending & $5(18.5)$ & $0.6(0.2-2.0)$ \\
\hline & Bar worker or owner & $2(7.4)$ & $2.3(0.4-14.3)$ \\
\hline & Waitress/Waiter/restaurant owner & $1(3.7)$ & $0.9(1.0-8.8)$ \\
\hline & Construction & $1(3.7)$ & $4.6(0.3-79.9)$ \\
\hline & Boda Boda & $1(3.7)$ & $1.5(0.1-16.3)$ \\
\hline
\end{tabular}

Pre-lockdown SARS-CoV-2 antibody test results: Upon initial screening using the CoronaChek ${ }^{\mathrm{TM}}, 7 \%$ $(47 / 363)$ of specimens had detectable antibodies to SARS-CoV-2 irrespective of isotype class. The majority (44) were IgM sole reactive, 2 were positive on IgG only whereas 1 reacted for both IgM and IgG. Out of the $44 \mathrm{IgM}$ sole positive samples, 8 were confirmed following re-testing using the ARCHITECT assay. The overall seroprevalence of antibodies to SARS-CoV-2 in this sample category was $1.7 \%$ [ $95 \% \mathrm{Cl}$ : 0.9-3.1] (11/636).

\section{Discussion}

These findings suggest a relatively high SARS-CoV-2 seroprevalence among HCWs at almost all the selected health facilities (24/26) in South-central Uganda and substantial seroprevalence in persons previously reporting specific COVID-19 like symptoms within the general population. There was also 
potentially a spike in transmission a few weeks prior to this evaluation with predominance of IgM only antibodies in most of the participants.

There are challenges interpreting SARS-CoV-2 rapid serology in regions with high malaria endemicity as infection with Plasmodium species was shown to induce cross-reactive antibodies to carbohydrate epitopes on the SARS-CoV-2 spike protein $(17,18)$. It is thus unclear whether seropositivity in prelockdown plasma specimens implies prior SARS-CoV-2 or other related coronavirus exposure or malaria in Uganda.

HCWs are minimally protected by face masks and only a few had accesses to other PPE (face shields, gowns, aprons etc.) and this, coupled with likelihood of improper face mask use or lack of N95-level protection, could explain the positive COVD-19 antibody results observed even among participants reporting face mask use. Several undetected cases among HCWs in this region is a potential driver of nosocomial spread. A moderate concordance between reported RT-PCR COVID-19 positives and antibody test outcome may reflect waning antibody levels as reported in several publications $(19,20)$.

\section{Conclusions}

Findings suggest a high seroprevalence of antibodies to SARS-CoV-2 among HCWs and substantial exposure in persons presenting with specific COVID-19 like symptoms in the general population of Southcentral Uganda. Based on current limitations in serological test confirmation, it remains unclear whether pre-lockdown seropositivity implies prior SARS-CoV-2 exposure in Uganda.

\section{Abbreviations}

SARS-CoV-2 Severe Acute Respiratory Syndrome Coronavirus 2

HCWs Healthcare workers

COVID-19 Coronavirus disease 2019

CMIA chemiluminescent microparticle immunoassay

WHO World Health Organization

PPE Personal Protective Equipment

UNCST Uganda National Council for Science and Technology

RCCS Rakai Community Cohort Study

RHSP Rakai Health Sciences Program

\section{Declarations}


Ethics approval and consent to participate: All methods of this study were carried out in accordance with the Uganda National Council for Science and Technology (UNCST) guidelines, the body that regulates research in Uganda. It was approved by the Uganda Virus Research Institute's Research Ethics Committee (Ref. GC/127/20/08/785), registered, and cleared by the UNCST (registration number HS878ES). Written informed consent was obtained from participants before blood specimens and other data were collected. Also, only archived pre-lockdown plasma specimens from Rakai Community Cohort Study (RCCS) participants that had provided prior written informed consent for use of their blood specimens in future studies were retrieved to assess prior SARS-CoV-2 exposure in Uganda. All participants were aged 18 years and above. No informed consent from 'Legally authorized representatives/parents' of minors below 16 years of age was thus required.

Consent for publication: Not applicable

Availability of data and materials: All data generated or analyzed during this study are included in this published article.

Competing interests: The authors declare that they have no competing interests.

Funding: This work was funded by the Government of Uganda through Makerere University Research and Innovations Fund (Grant number RIF/COVID/075, https://rif.mak.ac.ug/ ) and in part by the Division of Intramural Research, National Institute of Allergy, and Infectious Diseases (NIH, https://www.niaid.nih.gov/about/dir ). The funders had no role in study design, data collection and interpretation, or the decision to submit the work for publication.

Authors' contributions: Protocol development: RMG, DS, ENK, CS, TCQ, KMG, LWC, SJR, MJW, RHG; Study implementation: CS, RMG, DS, RS, JBW, AN, EM, MCN, SJ, JBO, AM, MA, MS, GC; Manuscript development: CS, DS, RMG, ENK, JK, GK, GN, TCQ, KMG, LWC, SJR, TL, MJW, RHG, OL

Acknowledgements: Samples/Data collection: RCCS field team, District Health Officers of Rakai, Kyotera, Lyantonde and Masaka districts, Prossy Namutebi, Wilson Bwanike; Data management: Damalie Nansimbi, Muhammed Mugerwa, Darix Ssebagala Kigozi

\section{References}

1. Lescure F-X, Bouadma L, Nguyen D, Parisey M, Wicky P-H, Behillil S, et al. Clinical and virological data of the first cases of COVID-19 in Europe: a case series. The Lancet Infectious Diseases. 2020.

2. Johns Hopkins University, cartographer JHU Corona Virus Resource Center. Baltimore, MD 21218, United States2020.

3. Meyerowitz EA, Richterman A, Gandhi RT, Sax PE. Transmission of SARS-CoV-2: a review of viral, host, and environmental factors. Annals of internal medicine. 2020.

4. Yanes-Lane M, Winters N, Fregonese F, Bastos M, Perlman-Arrow S, Campbell JR, et al. Proportion of asymptomatic infection among COVID-19 positive persons and their transmission potential: A 
systematic review and meta-analysis. PloS one. 2020;15(11):e0241536.

5. Arons MM, Hatfield KM, Reddy SC, Kimball A, James A, Jacobs JR, et al. Presymptomatic SARS-CoV2 infections and transmission in a skilled nursing facility. New England journal of medicine. 2020.

6. Nikolai LA, Meyer CG, Kremsner PG, Velavan TP. Asymptomatic SARS Coronavirus 2 infection: Invisible yet invincible. International Journal of Infectious Diseases. 2020.

7. Furukawa NW, Brooks JT, Sobel J. Evidence supporting transmission of severe acute respiratory syndrome coronavirus 2 while presymptomatic or asymptomatic. Emerging infectious diseases. 2020;26(7).

8. B. Oketch., P. Ebong., R. Muhereza., A. Ssenkabirwa., M.F. Jjingo., P. Kalokwera., et al. Health facilities run out of space as Covid-19 cases rise. Daily Monitor. 2020.

9. P. Ahimbisibwe. Museveni orders two-week lockdown as COVID-19 cases rise. Daily Monitor. 2020.

10. Ran L, Chen X, Wang Y, Wu W, Zhang L, Tan X. Risk factors of healthcare workers with corona virus disease 2019: a retrospective cohort study in a designated hospital of Wuhan in China. Clinical Infectious Diseases. 2020.

11. Wang $X$, Jiang $X$, Huang $Q$, Wang H, Gurarie D, Ndeffo-Mbah M, et al. Risk factors of SARS-CoV-2 infection in healthcare workers: a retrospective study of a nosocomial outbreak. Sleep Medicine: X. 2020;2:100028.

12. Papoutsi E, Giannakoulis VG, Ntella V, Pappa S, Katsaounou P. Global burden of COVID-19 pandemic on healthcare workers. Eur Respiratory Soc; 2020.

13. Twinamukye P. Uganda loses 58 doctors, nurses within one year. Daily Monitor 2021.

14. Chang LW, Mbabali I, Kong X, Hutton H, Amico KR, Kennedy CE, et al. Impact of a community health worker HIV treatment and prevention intervention in an HIV hotspot fishing community in Rakai, Uganda (mLAKE): study protocol for a randomized controlled trial. Trials. 2017;18(1):1-12.

15. Baker OR, Grabowski MK, Galiwango RM, Nalumansi A, Serwanga J, Clarke W, et al. Differential Performance of CoronaCHEK SARS-CoV-2 Lateral Flow Antibody Assay by Geographic Origin of Samples. Journal of Clinical Microbiology. 2021:JCM. 00837-21.

16. Woodford J, Sagara I, Kwan J, Zeguime A, Zaidi I, Attaher O, et al. SARS-CoV-2 seroassay optimization and performance in a population with high background reactivity in Mali. 2021.

17. Lapidus S, Liu F, Casanovas-Massana A, Dai Y, Huck JD, Lucas C, et al. Plasmodium infection induces cross-reactive antibodies to carbohydrate epitopes on the SARS-CoV-2 Spike protein. medRxiv. 2021.

18. Grifoni A, Weiskopf D, Ramirez SI, Mateus J, Dan JM, Moderbacher CR, et al. Targets of T cell responses to SARS-CoV-2 coronavirus in humans with COVID-19 disease and unexposed individuals. Cell. 2020;181(7):1489-501. e15.

19. Choe PG, Kang CK, Suh HJ, Jung J, Song K-H, Bang JH, et al. Waning Antibody Responses in Asymptomatic and Symptomatic SARS-CoV-2 Infection. Emerg Infect Dis. 2020;27. 
20. Perreault J, Tremblay T, Fournier M-J, Drouin M, Beaudoin-Bussières G, Prévost $J$, et al. Waning of SARS-CoV-2 RBD antibodies in longitudinal convalescent plasma samples within 4 months after symptom onset. Blood, The Journal of the American Society of Hematology. 2020;136(22):2588-91. 